\title{
Schwannoma of the Kidney
}

\author{
Stefano Gobbo ${ }^{1,2}$, John N Eble ${ }^{1}$, Jiaoti Huang ${ }^{3}$, David J Grignon ${ }^{1}$, Mingsheng Wang ${ }^{1}$, \\ Guido Martignoni ${ }^{2}$, Matteo Brunelli ${ }^{2}$ and Liang Cheng ${ }^{1}$ \\ ${ }^{1}$ Department of Pathology and Laboratory Medicine, Indiana University, Indianapolis, IN, USA; \\ ${ }^{2}$ Dipartimento di Patologia, Universitá di Verona, Verona, Italy and ${ }^{3}$ Department of Pathology, University of \\ Rochester Medical Center, Rochester, NY, USA
}

\begin{abstract}
Schwannomas of the kidney are rare, with only a few reported cases. We report three additional cases with immunohistochemical analysis. All three tumors were from females (aged 27, 35, and 59 years) and ranged from 4.8 to $8 \mathrm{~cm}$ in diameter. All of the patients underwent nephrectomy. The tumors were totally or partially encapsulated; two were in the hilum and one was centered in the renal cortex. All tumors were diffusely positive for S100 protein. Two were positive for neuron-specific enolase. Immunostaining for neurofilament, HMB45, microphthalmia transcription factor, smooth muscle actin, CD34, cytokeratin AE1/3, cytokeratin 7, and CD10 were negative. Follow-up data were available for two patients; neither had tumor recurrence or metastasis. In conclusion, renal schwannoma is rare, usually arises centrally, impinging on the hilum or the pelvis, and is cured by resection. Sarcomatoid carcinoma and other spindle cell tumors should be considered in the differential diagnosis.

Modern Pathology (2008) 21, 779-783; doi:10.1038/modpathol.2008.52; published online 4 April 2008
\end{abstract}

Keywords: kidney; neoplasia; soft tissue tumor; schwannoma; biomarker; immunohistochemistry

Schwannomas are uncommon tumors that originate from the neural sheath and are most common in the head and neck, extremities, and posterior mediastinum. Although $3 \%$ of schwannomas occur in the retroperitoneum, involvement of visceral organs is extremely uncommon. ${ }^{1}$ Schwannomas are rare in the kidneys, and only 19 cases have been reported. ${ }^{2-16}$ We report herein the clinicopathologic features of three additional cases of renal schwannoma.

\section{Materials and methods}

Clinical findings, operative data, and patient followup were obtained from medical records. Immunohistochemical preparations were performed on formalin-fixed, paraffin-embedded tissue in all cases. The antibodies, clones, dilutions, antigen retrieval methods, and vendors are listed in Table 1. Appropriate positive and negative controls for each antibody were run concurrently.

Correspondence: Dr L Cheng, MD, Department of Pathology and Laboratory Medicine, Indiana University School of Medicine, 350 West 11th Street, Clarian Pathology Laboratory Room 4010, Indianapolis, IN 46202, USA.

E-mail: liang_cheng@yahoo.com

Received 04 November 2007; revised 28 January 2008; accepted 05 February 2008; published online 4 April 2008

\section{Results}

Clinical findings are summarized in Table 2. The patients were all women and were 27,35 , and 59 years old. None showed any sign of neurofibromatosis type 1. Clinical presentations included abdominal pain and nausea in one case; the other two patients were asymptomatic. Imaging revealed a renal mass suspicious for renal cell carcinoma in each case. Subsequently, two patients underwent radical nephrectomy and one had a laparoscopic nephrectomy. Short-term (4 and 8 months) followup data were available from two patients. Both were alive without recurrence or metastasis.

Macroscopically, case 1 showed a $4.8 \times 4.2 \times$ $4.2 \mathrm{~cm}$ well-circumscribed lobulated nodular lesion located in the hilum, compressing the renal pelvis, and distorting the surrounding vasculature. Its cut surface was gray-tan to yellow and fibrotic. Case 2 showed an $8.5 \times 5.5 \times 5 \mathrm{~cm}$ well-circumscribed, red-brown fibrous mass centered in the renal cortex in the middle and lower pole of the kidney. Its cut surface appeared myxomatous and fibrous. Case 3 showed a $7 \times 4.8 \times 4 \mathrm{~cm}$ multicystic, encapsulated, focally gelatinous-appearing mass involving the hilum of the kidney.

Histologically, all three tumors were encapsulated and showed characteristic features of schwannoma. In cases 1 and 3 , the tumor was composed mainly of spindle cells with a loose textured pattern (Antoni B) and with microcyst formation, and some cellular 
Table 1 Antibody panel used in this study

\begin{tabular}{lllll}
\hline Antibody/molecule & Clone & Dilution & Antigen retrieval & Source (city/state) \\
\hline S100 & Polyclonal & $1: 100$ & Dako pH 6.0 citrate & Dako (Carpenteria, CA, USA) \\
NSE & BBS/NC/V1-H14 & Prediluted & Dako pH 6.0 citrate & NeoMarker (Fremont, CA, USA) \\
Neurofilament & 2F11 & $1: 500$ & EDTA pH 8.0 & Cell Marque (Rocklin, CA, USA) \\
HMB45 & HMB-45 & $1: 30$ & Dako pH 6.0 citrate & Dako (Carpenteria, CA, USA) \\
MiTF & D5 & $1: 100$ & Dako pH 6.0 citrate & Dako (Carpenteria, CA, USA) \\
SMA & 1A4 & Prediluted & None & Dako (Carpenteria, CA, USA) \\
CK AE1/3 & AE1/AE3 & $1: 80$ & Dako pH 6.0 citrate & Dako (Carpenteria, CA, USA) \\
CK 7 & OV-TL 12/30 & Prediluted & Proteinase K & Dako (Carpenteria, CA, USA) \\
CD34 & QBEND & Prediluted & Dako pH 6.0 citrate & Dako (Carpenteria, CA, USA) \\
CD10 & 56C6 & Prediluted & Borg pH 9.0 & Cell Marque (Rocklin, CA, USA)
\end{tabular}

CK, cytokeratin; NSE, neuron-specific enolase; SMA, smooth muscle actin.

areas with Verocay bodies (Antoni A). In case 2, the tumor showed extensive hyaline change in a predominantly Antoni B pattern composed of pleomorphic cells with smudged-appearing chromatin, consistent with degenerative changes. Vascular sclerosis, hemorrhage, and hemosiderin deposits were observed in case 3 . Isolated or scattered nuclear atypia was present in cases 2 and 3 . Rare mitotic figures were detected in case 1 . Necrosis was not observed (Figure 1).

In each tumor, the neoplastic cells showed strong and diffuse immunoreactivity for S100 protein. Neuron-specific enolase was expressed in about $90 \%$ of the cells in cases 2 and 3 and in $10 \%$ of the cells in case 1. CD34 was expressed only in the endothelial cells. All other immunohistochemical reactions (neurofilament protein, cytokeratins (CK7 and $\mathrm{AE}$ 1/3), HMB45, microphthalmia transcription factor, smooth muscle actin, and CD10) were negative.

\section{Discussion}

Schwannomas consist of a nearly pure population of cells showing schwannian differentiation, and arise eccentrically from the surface of their nerve of origin. The main nerves of the kidney consist of sympathetic and parasympathetic fibers that accompany the renal artery entering in the renal hilum. This could explain the more frequent location of schwannomas at the renal hilum. Schwannoma rarely arises from the kidneys with only 19 cases reported (Table 2). ${ }^{2-16}$ Clinical presentations include abdominal or flank pain (12 cases), ${ }^{2,4-6,8,10,11,13}$ microhematuria (2 cases), ${ }^{3,15}$ or fever (4 cases). ${ }^{4,6,10,11}$ Sometimes patients are asymptomatic and the finding is incidental. ${ }^{2,12,14,16}$ No patient has been reported to show any sign of neurofibromatosis type 1. All patients are adults and the mean age at diagnosis is 51 years (range, 18-84 years). The maleto-female ratio is $1: 1.2$. The mass is often located centrally in the kidney, impinging on the hilum and renal pelvis. Less commonly, the tumors appear to arise from the renal cortex or capsule. Two previously reported cases (9\%) were diagnosed as 'malignant schwannomas' and those patients died with metastases 3 and 15 months after the diagnosis. ${ }^{9,11}$ These cases probably represent malignant peripheral nerve sheath tumor since rarely does malignancy truly arise in a benign schwannoma.

The histologic appearance of schwannoma of the kidney is similar to that seen in other organs and includes the range of different morphologic patterns described in schwannomas. The classic schwannoma is characterized by a mixture of Antoni A and Antoni B patterns as seen in cases 1 and $3 .{ }^{17}$ Microcystic degeneration with hemorrhage and hemosiderin deposits associated with cells showing bizarre hyperchromatic nuclei are common degenerative features of large schwannomas usually seen in retroperitoneum. ${ }^{18}$ The tumor in case 2 showed the features of the so-called ancient schwannoma. Ancient schwannomas are not considered as a distinct subtype of schwannoma, but rather longstanding tumors with degenerative nuclear and architectural changes. They do not seem to differ clinically from conventional schwannomas. ${ }^{19}$ Cellular schwannoma is exclusively composed of spindle cells in the Antoni A pattern without Verocay bodies, ${ }^{17,20}$ a pattern described in four of the reported renal schwannomas. ${ }^{2,8}$

Differential diagnostic considerations include low-grade malignant peripheral nerve sheath tumor, renal angiomyolipoma, sarcomatoid carcinoma, synovial sarcoma, solitary fibrous tumor, leiomyoma, low-grade leiomyosarcoma, rhabdomyosarcoma, and angiosarcoma. The distinctive clinical, histologic, and immunohistochemical findings usually permit a definitive diagnosis.

S100 protein family is the largest subgroup of the EF-hand proteins, which consists of several members that display amino-acid sequence homology ranging from 25 to $65 \%$. S100 protein has been studied in the spectrum of epithelial tumors of the kidney and has been proposed to be a useful tool in the differential diagnosis among renal cell neoplasms, particularly in the differential diagnosis of chromophobe renal cell carcinoma vs renal oncocytoma. $^{20}$ Among renal tumors, S100 protein is 
Table 2 Clinicopathologic findings of three cases of renal schwannoma and review of the literature

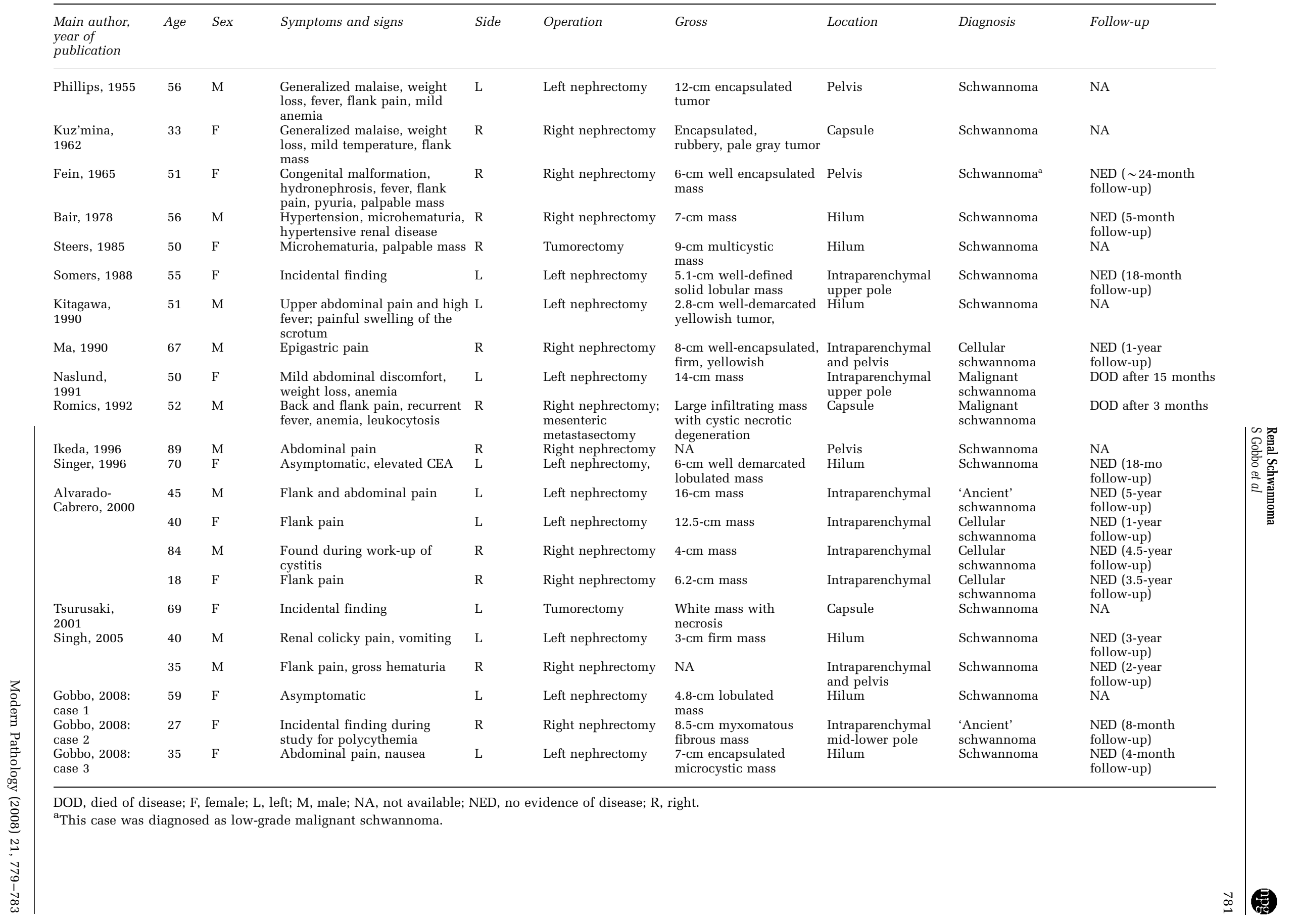



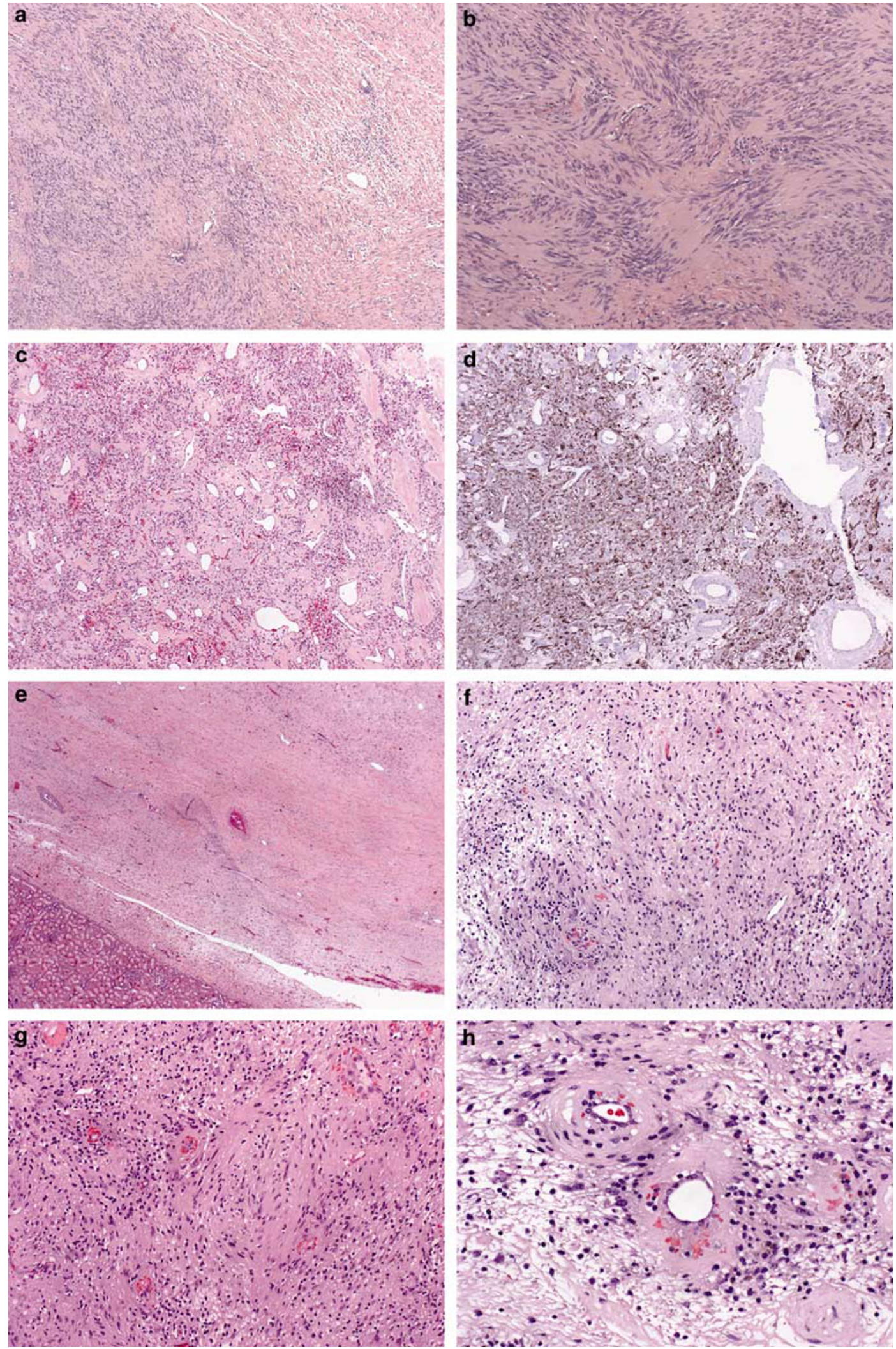

Figure 1 Schwannoma of the kidney; (a) (case 1) the tumor is characterized by a mixture of Antoni A and Antoni B pattern; (b) (case 1) cellular area with Verocay bodies (Antoni A); (c) (case 2) the tumor showed extensive hyaline change; (d) (case 2) neoplastic cells showed a strong and diffuse immunoreactivity with S100 protein; (e) (case 3) the tumor was separated from the renal parenchyma by a wellformed fibrous capsule; (f) (case 3) mixture of Antoni A and Antoni B patterns in a renal schwannoma; (g) (case 3) schwannoma showing vascular sclerosis; (h) (case 3) Antoni B area showing vascular sclerosis and hemosiderin deposits. 
expressed in oncocytoma, papillary renal cell carcinoma, and clear cell renal cell carcinoma, whereas they are negative in chromophobe renal cell carcinoma. ${ }^{20}$ In the current study, all three cases of renal schwannoma were positive for S100 protein.

Schwannomas rarely undergo malignant change, most often in the form of either malignant peripheral nerve sheath tumor or angiosarcoma. ${ }^{21-23}$ Malignant peripheral nerve sheath tumor is occasionally composed of epithelioid cells with vesicular nuclei and prominent nucleoli. ${ }^{24}$ Angiosarcoma is typically immunoreactive for endothelial markers, such as CD34. ${ }^{22}$ The presence of nuclear atypia or diffuse hypercellularity in a schwannoma may lead to an erroneous diagnosis of malignancy. It is critical to distinguish schwannoma of atypical or cellular type from malignant peripheral nerve sheath tumor. Unlike malignant peripheral nerve sheath tumors, schwannomas with degenerative nuclear atypia lack mitotic figures. Their large cells with pleomorphic nuclei and smudged chromatin are reminiscent of those seen in ancient schwannoma. Cellular schwannoma is distinguished from malignant peripheral nerve sheath tumor by the smaller cell size, infrequent mitotic figures and lack of significant cytologic atypia, nuclear pleomorphism, and necrosis. In the absence of definite cellular crowding, nuclear enlargement $(\geq 3$ times the size of ordinary schwannoma nuclei) and hyperchromasia, the finding of rare mitotic figures in a cellular schwannoma is not sufficient for a diagnosis of malignancy.

In summary, schwannoma rarely arises from the kidney, is usually located in the renal hilum or pelvis, and clinically behave in a benign fashion.

\section{References}

1 Gubbay AD, Moschilla G, Gray BN, et al. Retroperitoneal schwannoma: a case series and review. Aust N Z J Surg 1995;65:197-200.

2 Alvarado-Cabrero I, Folpe AL, Srigley JR, et al. Intrarenal schwannoma: a report of four cases including three cellular variants. Mod Pathol 2000;13: 851-856.

3 Bair ED, Woodside JR, Williams WL, et al. Perirenal malignant Schwannoma presenting as renal cell carcinoma. Urology 1978;11:510-512.

4 Fein RL, Hamm FC. Malignant schwannoma of the renal pelvis: a review of the literature and a case report. J Urol 1965;94:356-361.

5 Ikeda I, Miura T, Kondo I, et al. Neurilemmoma of the kidney. Br J Urol 1996;78:469-470.
6 Kitagawa K, Yamahana T, Hirano S, et al. MR imaging of neurilemoma arising from the renal hilus. J Comput Assist Tomogr 1990;14:830-832.

7 Kuz'Mina VE. [Neurinoma of the kidney capsule.]. Urol Mosc 1962;27:52-53.

8 Ma KF, Tse CH, Tsui MS. Neurilemmoma of kidney-a rare occurrence. Histopathology 1990;17:378-380.

9 Naslund MJ, Dement S, Marshall FF. Malignant renal schwannoma. Urology 1991;38:477-479.

10 Phillips CA, Baumrucker G. Neurilemmoma (arising in the hilus of left kidney). J Urol 1955;73:671-673.

11 Romics I, Bach D, Beutler W. Malignant schwannoma of kidney capsule. Urology 1992;40:453-455.

12 Singer AJ, Anders KH. Neurilemoma of the kidney. Urology 1996;47:575-581.

13 Singh V, Kapoor R. Atypical presentations of benign retroperitoneal schwannoma: report of three cases with review of literature. Int Urol Nephrol 2005;37: 547-549.

14 Somers WJ, Terpenning B, Lowe FC, et al. Renal parenchymal neurilemoma: a rare and unusual kidney tumor. J Urol 1988;139:109-110.

15 Steers WD, Hodge GB, Johnson DE, et al. Benign retroperitoneal neurilemoma without von Recklinghausen's disease: a rare occurrence. J Urol 1985;133:846-848.

16 T1surusaki M, Mimura F, Yasui N, et al. Neurilemoma of the renal capsule: MR imaging and pathologic correlation. Eur Radiol 2001;11:1834-1837.

17 Kurtkaya-Yapicier O, Scheithauer B, Woodruff JM. The pathobiologic spectrum of Schwannomas. Histol Histopathol 2003;18:925-934.

18 Weiss SW, Goldblum JR. Enzinger and Weiss's Soft Tissue Tumors, 5th edn. Mosby: Philadelphia, PA, 2008, pp 825-902.

19 Scheithauer BW, Woodruff JM, Erlandson RA. Tumors of the Peripheral Nervous System. American Forces Institute of Pathology: Washington, DC, 1999.

20 Martignoni G, Brunelli M, Gobbo S, et al. Role of molecular markers in diagnosis and prognosis of renal cell carcinoma. Anal Quan Cytol Histol 2007;29:41-49.

21 Mikami Y, Hidaka T, Akisada T, et al. Malignant peripheral nerve sheath tumor arising in benign ancient schwannoma: a case report with an immunohistochemical study. Pathol Int 2000;50:156-161.

22 Trassard M, Le Doussal V, Bui BN, et al. Angiosarcoma arising in a solitary schwannoma (neurilemoma) of the sciatic nerve. Am J Surg Pathol 1996;20:1412-1417.

23 Woodruff JM, Selig AM, Crowley K, et al. Schwannoma (neurilemoma) with malignant transformation. A rare, distinctive peripheral nerve tumor. Am J Surg Pathol 1994;18:882-895.

24 McMenamin ME, Fletcher CD. Expanding the spectrum of malignant change in schwannomas: epithelioid malignant change, epithelioid malignant peripheral nerve sheath tumor, and epithelioid angiosarcoma: a study of 17 cases. Am J Surg Pathol 2001;25:13-25. 